Meta

Journal des traducteurs

Translators' Journal

\title{
La stylistique française enseignée à des apprentis-traducteurs
}

\section{Colette Touitou-Benitah}

Volume 36, numéro 2-3, juin 1991

URI : https://id.erudit.org/iderudit/002055ar

DOI : https://doi.org/10.7202/002055ar

Aller au sommaire du numéro

Éditeur(s)

Les Presses de l'Université de Montréal

ISSN

0026-0452 (imprimé)

1492-1421 (numérique)

Découvrir la revue

Citer cet article

Touitou-Benitah, C. (1991). La stylistique française enseignée à des

apprentis-traducteurs. Meta, 36(2-3), 456-460. https://doi.org/10.7202/002055ar d'utilisation que vous pouvez consulter en ligne.

https://apropos.erudit.org/fr/usagers/politique-dutilisation/ 


\section{LA STYLISTIQUE FRANÇAISE ENSEIGNÉE À DES APPRENTIS- TRADUCTEURS}

Colette Touttou-Benitah

Université Bar-Ilan, Ramat Gan, Israël

\section{DONNÉES}

Cet enseignement s'adresse à des étudiants de première année de l'École de traducteurs-interprètes de l'Université Bar-Ilan en Israël. Ces étudiants sont, soit francophones de naissance, et se destinent à devenir des traducteurs dont la langue d'arrivée sera le français; soit des étudiants dont le niveau de français a été jugé satisfaisant à la suite d'examens d'entrée, et dont le français sera la langue de départ lors de l'exercice de leur métier futur.

Les uns comme les autres ont préalablement achevé une licence de lettres; dans la plupart des cas, une licence de langues.

Face à ce groupe constitué d'apprenants le français langue maternelle, et d'apprenants le français langue étrangère 1 , un des premiers soucis a été de ne pas sous-estimer l'acquis des uns et de ne pas surestimer les compétences des autres. Le problème était, désormais, de passer des intentions aux actes et de fonder cet enseignement sur une théorie et sur une méthode appropriées qui permettent aux premiers de ne pas être inhibés par la présence des francophones, et aux seconds de prendre conscience de leurs limites; puis, à partir de là, de dépasser la situation donnée et d'enrichir leurs connaissances respectives.

\section{BASES THÉORIQUES ET PRAGMATISME}

Il convient tout d'abord de préciser que la stylistique enseignée à des apprentistraducteurs doit être cette «étude systématique des moyens d'expression» dont parle Charles Bally ${ }^{2}$. Cette stylistique n'est pas une stylistique du sujet parlant en ce qu'il a de particulier et de personnel, mais plutôt une stylistique de règles et de combinatoires des sujets parlants. Non pas une stylistique fondée sur une conception du style en tant qu'écart par rapport à une norme ${ }^{3}$, mais au contraire une stylistique pour qui le style est ce langage naturel, ce fait d'expression dont il convient d'étudier la nature. Une stylistique qui analyse le discours en intégrant la dimension psychologique du langage et en la subjugant à sa dimension sociale, elle-même coulée dans le moule de la linguistique. Une stylistique enfin qui doit aboutir nécessairement à une typologie des discours.

Par ailleurs, dans l'objectif professionnel, l'utilisation du français devant être pour les étudiants FLM une utilisation de la langue écrite (traduction de l'hébreu au français) - alors que pour les étudiants FLE, le français est exclusivement une langue de lecture (traduction du français à l'hébreu) - il fallait par un même enseignement viser à développer les performances écrites chez les FLM, et former les FLE à une sensibilité accrue au français, base d'une lecture digne de ce nom. Comment, par un même enseignement, former des lecteurs attentifs et des rédacteurs exigeants?

La nécessité d'un travail conjoint des deux groupes, dictée par des contraintes extralinguistiques, a impliqué une coopération constante. Ces donnés impératives ont eu pour conséquence le besoin de fonder cet enseignement sur des bases théoriques et 
pragmatiques empruntées aux deux cultures, aussi bien à la culture occidentale dans son approche de la langue, telle qu'elle apparaît dans les sciences du langage qu'à la culture hébraïque, dans sa tradition de l'étude.

Recréant la situation de communication, telle que Jakobson 1'a décrite ${ }^{4}$, les FLM se sont trouvés en position d'émetteurs, tandis que les FLE étaient eux les récepteurs. Une telle coopération devait conférer aux deux groupes un statut différent mais non dépréciatif.

À partir de là, la scission naturelle et linguistique entre groupe FLM et groupe FLE était évitée et à sa place pouvait s'instaurer une organisation par petits groupes de deux étudiants, un FLM et un FLE, un émetteur et un récepteur, groupuscules dynamiques favorisant des échanges linguistiques fructueux.

Cette étude à deux a été d'autant mieux acceptée qu'elle s'inscrit dans l'antique tradition de l'étude talmudique juive ${ }^{5}$. En effet, depuis plus de deux mille ans et jusqu'à ce jour, en Israël et dans la Diaspora, dans les Yechivot ou Instituts d'études talmudiques, les étudiants ne sont pas seuls face à leur livre, mais ils sont groupés par deux, dans un face à face constant, dans une relation je-tu où la parole des Sages consignée dans les livres constitue une troisième personne, sans cesse discutée 6 .

Ainsi l'ancien et le moderne s'alliaient pour fournir les bases d'un enseignement de la stylistique française à des apprentis-traducteurs, à qui il importait de faire comprendre d'emblée que le perfectionnement de la connaissance d'une langue dans l'optique de la traduction ne peut se faire sans la connaissance sympathique, sympathique pris dans le sens étymologique, des deux cultures.

\section{ANALYSE DE L'ÉNONCIATION, EN GUISE DE PRÉLUDE}

Le traducteur est le récepteur d'un message inséré dans une langue-culture, et l'émetteur de ce même message inséré dans une autre langue-culture. Il n'est pas un simple bi-lingue ${ }^{7}$. Sa lecture de l'énoncé est une lecture énonciative qui réintègre le je-icimaintenant, c'est-à-dire le sujet de l'énonciation, dans son micro-contexte psychologique, et dans son macro-contexte sociologique, culturel. Le traducteur est ce réénonciateur ${ }^{8}$ qui réinsère le micro-contexte dans un nouveau macro-contexte.

Bien que l'enseignement du français dans le cadre strict de ce cours ne comporte pas de pratique de la traduction, il se doit de se situer par rapport à la traduction, et ne peut ignorer les mécanismes mentaux du traducteur.

C'est la raison pour laquelle il a semblé adéquat de fonder cet enseignement sur une méthodologie de l'énonciation, d'autant plus que notre École a pour vocation de former des traducteurs-interprètes aptes à traduire des discours aussi bien littéraires que scientifiques ou techniques.

Ce cours, dans les limites horaires d'une heure et demie d'enseignement hebdomadaire pendant deux semestres universitaires, a été consacré au cours du premier semestre à l'étude systématique des différentes parties du discours et à des exercices de correction de style. Par correction de style, il faut entendre une meilleure adéquation de l'énoncé aux conditions de l'énonciation. Cette adéquation est très souvent problématique pour les étudiants FLE $^{9}$. Ceux-ci ont tendance, particulièrement dans le cadre de l'apprentissage de la traduction professionnelle, à vouloir en tout premier lieu élargir leur lexique. Or ce n'est pas suffisant. Il est urgent de développer chez eux la conscience de l'interaction entre énoncé et énonciation. C'est à partir d'exercices appropriés, et également à partir des fautes des apprenants, aussi bien à l'écrit qu'à l'oral, que la correction de style peut être productive et mener l'étudiant à une meilleure adéquation de ses énoncés. L'enseignant, quant à lui, doit être capable d'analyser avec finesse ces fautes afin de transformer la correction de style en un stade d'apprentissage important où la faute devient le levier du progrès et de l'enrichissement. Le second semestre devait, lui, être consacré à l'étude 
des différents types de discours ${ }^{10}$, et a été organisé autour d'un double type de corpus ${ }^{11}$, constitué ainsi.

Le premier type de corpus, constitué de textes généralement courts, empruntés aux divers types de discours, a fait l'objet d'analyses de l'énonciation réalisées en cours. Ces analyses, basées sur l'étude minutieuse des référents personnels et de leurs substituts, ainsi que sur celle des référents spatio-temporels, de la prédétermination, des modalisations, des transformations syntaxiques ${ }^{12}$, constituent une formation de base pour l'apprentitraducteur.

À l'intérieur de ce corpus, l'étude de certains types de discours qui se traduisent plus que d'autres sera plus approfondie. Si la moyenne du nombre des séances consacrées à l'étude d'un type de discours est de deux, le discours publicitaire, qui ne se traduit pas vraiment à cause de la prépondérance de son appareil référentiel ${ }^{13}$, n'occupera qu'une séance, alors que celle du discours politique ou juridique pourra être étudiée pendant trois à quatre séances 14 .

Le second corpus, constitué d'un ensemble d'articles de la presse quotidienne ou hebdomadaire comme le Monde, l'Express, le Nouvel Observateur, a fourni un corpus informatif actualisé sur la culture française, tandis qu'un autre ensemble constitué d'articles de réflexion sur la France et l'Europe en général était fourni par des périodiques telles les revues Commentaire, Europe, etc.

Ces textes à lire et à travailler par groupe de deux, un FLE, un FLM, en dehors des cours, font l'objet d'un approfondissement de la langue française et des deux cultures française et israélienne. En effet, pour chaque texte, l'étudiant FLE doit remettre une fiche de préparation comprenant une liste de mots nouveaux expliqués ainsi qu'un relevé des connotations culturelles que son camarade FLM l'aura aidé à détecter et à comprendre. La fiche de travail de l'étudiant FLM doit comporter, elle, soit une contraction de texte, soit une dissertation sur le thème central de l'article. Chaque étude de type de discours donne enfin lieu à un travail commun aux deux étudiants et permet à l'étudiant FLE d'être cette fois-ci l'émetteur tandis que son camarade FLM est le récepteur, puisqu'il s'agit d'étudier pour chaque type de discours la réalité extra-linguistique qui sous-tend ce discours dans la culture hébraïque ou la société israélienne ${ }^{15}$.

\section{AU COMMENCEMENT, RECOMMENCEMENTS}

L'enseignement de la stylistique française dans l'optique de la traduction professionnelle et dans les conditions toutes particulières de ce cours commun à des apprenants FLE, FLM, pose plus d'un problème et est riche en défis pour l'enseignant, qui, attentivement à l'écoute de deux cultures, doit sans cesse s'adapter et vivre la langue sur le mode dialectique. L'exégèse biblique juive, dans sa sagesse, explique que le premier mot du récit de la création du monde commence par la deuxième lettre de l'alphabet hébraique pour nous enseigner que tout, en ce monde, est duel.

Piège ou défi, cette dualité sera-t-elle ferment de progrès ou non? Tout dépendra de la réflexion et du travail investis dans l'enseignement de la traduction qui est, sans conteste, un formidable chantier où le contact avec l'autre langue, l'autre culture, voulu, assumé, valorisé, est certainement source d'enrichissement mutuel.

La répartition FLM/FLE à parts égales n'étant pas toujours le cas, les stratégies d'enseignement décrites ci-dessus sont à adapter.

Néanmoins, d'année en année, de commencement en recommencement, l'analyse de l'énonciation et ses techniques acquises lors de ce cours de stylistique ont prouvé qu'elles constituaient une base sûre, stable, et assuraient une continuité d'approche et de méthode entre ce cours et le cours de traduction conçu comme l'apprentissage du passage de l'énonciation à la réénonciation. 


\section{NOTES}

1. Désignés ultérieurement par les sigles FLM (français langue maternelle) et FLE (français langue étrangère).

2. Il est particulièrement intéressant pour nous de noter que c'est à la suite de leçons de traduction d'allemand en français, données dans les années 1890 à Genève, que Charles Bally met au point une théorie nouvelle du langage. Voir José Médina (1985): «Charles Bally: de Bergson à Saussure», Langages, $\mathrm{n}^{\circ} 77, \mathrm{pp}$. 95-104.

3. Cette conception du style, héritée de la tradition rhétorique classique, rejoint la tradition omementale du style telle qu'elle apparaît à partir des XVIe et XVIIe siècles dans les répertoires des ornements du discours tel le Traité des Tropes de Dumarsais (1729) et le Commentaire raisonné qu'en fait en 1818 Pierre Fontanier.

Voir Dumarsais-Fontanier (1967): «Les Tropes» avec une introduction de Gérard Genette, Genève, Slatkine Reprints.

La question de la conception du style n'a pas fini de préoccuper notre génération. Georges Molinié (1986), dans Éléments de stylistique française, PUF, dit précautionneusement: «le style paraît désigner une dynamique dans l'activité langagière, plutôt qu'un écart, une manière ou une qualité» (p. 145).

Pour approfondir cette notion, voir: Nicole Gueunier (1963): «La pertinence de la notion d'écart en stylistique», Langue française, $n^{\circ}$ 3. Jean-Claude Chevalier (1981): «Notes sur l'écart» L'enseignement de la littérature, Actes du colloque de Cerisy, A. de Bock et Duculot.

4. Roman Jakobson (1963): Essais de linguistique générale, les Éditions de Minuit. Évidemment, la réversibilité de la situation de communication, envisagée aussi bien par la linguistique moderne que par la pédagogie hébraïque traditionnelle, devait également faire des FLE des émetteurs, et des FLM des récepteurs. Dans ce cas, le message a été choisi dans le champ culturel des FLE pour leur permettre de concentrer leur attention sur leurs performances linguistiques en français.

5. De même que les anthropologues (Edgar Morin et Serge Moscovici en France, Robin Fox et Lionel Tiger aux U.S.A.) nous apprennent que dans l'antiquité, la chasse, activité collective, a permis le développement plus rapide de l'intelligence des hommes, alors que la cueillette, individuelle, a maintenu les femmes dans une espèce de sous-culture; les Sages d'Israël ont toujours estimé qu'il était dangereux d'étudier seul. L'étude à deux, appelée Havrouta, permet à l'élève d'aiguiser son intelligence au contact de l'autre, alors que l'étude individuelle, sans discussions, sans débats, sans argumentation et contre-argumentation «ne connaîtra pas la bénédictions.

Le système pédagogique de la Havrouta, outre le fait qu'il crée les conditions d'échanges intellectuels incessants, est un puissant facteur de socialisation. Les partenaires apprennent la coopération, la solidarité, et à un degré plus haut encore, la modestie inhérente à la conscience claire de leur propre valeur; toutes qualités que nous sommes intéressés à développer chez nos apprentis-traducteurs.

À propos de la Havrouta comme méthode d'étude, voir les nombreuses références dans le Talmud: Traités Avoth 1-6, Macoth 10-1, Taanith 7-1, Chabath 63-3, Berechith Rabba 69-2 cités par Moshe Averbach (1982): Ha-hinukh ha-yehudi bi-tkufat ha-Mishna we-ha-Talmud, Jérusalem, Rubin Mass. (Que le professeur Dov Rapel qui $m$ 'a aidée dans ma recherche sur la Havrouta soit jci remercié.)

6. Émile Benveniste (1966), dans ses articles «Structure des relations de personne dans le verbe» et «La nature des pronoms» (Problèmes de linguistique générale, Gallimard), prend comme point de départ la grammaire arabe pour mettre en lumière la disparité entre la $3 \mathrm{e}$ personne et les deux premières personnes. La grammaire hébraïque, comme la grammaire arabe, appelle la $1^{\text {re }}$ et la $2^{\mathrm{e}}$ personnes «celui qui parle» et «celui qui est présent», alors que la $3 e$ personne est «celui qui est caché». Les deux premières personnes, réversibles, réalisent la situation de communication. La $3^{e}$ personne, elle, est pour Benveniste cette «nonpersonne» qui a pour propriété de «n'être jamais réflexive de l'instance du discours». Le statut d'objet invariant qui est ainsi conféré au texte étudié, que ce soit une page de Talmud ou un article de presse, privilégie l'échange entre les apprenants.

7. Pour un approfondissement de la question, voir Jean Delisle (1984): L'analyse du discours comme méthode de traduction, Éditions de l'Université d'Ottawa, pp. 34-38.

8. La traduction comme réénonciation a été étudiée dans notre thèse pour le doctorat de 3e cycle (1978), Paris VIII. «L'expression de la relation à l'enfant dans deux nouvelles de Abraham B. Yehoshua. Étude de l'énonciation dans l'original hébraïque et de la réénonciation dans la traduction française» (texte ronéoté). Voir également l'entretien avec Antoine Culioli (1987): «Un point de vue énonciatif sur la traduction» in Le français dans le monde. Recherches et applications, numćro spécial «Retour à la traduction».

9. Ces exercices d'adéquation de l'énoncé à l'énonciation donnent lieu également à des exercices de paraphrase variés. À partir de conditions d'énonciation données, il s'agit de produire le plus grand nombre possible d'énoncés paraphrastiques adéquats, puis en modifiant une à une et de façon la plus fine qui soit les conditions d'énonciation, produire d'autres énoncés. On parvient ainsi à développer chez les FLE et à affiner chez les FLM la capacité de production d'énoncés sensibles aux plus petites variations de l'énonciation. Nombre d'exercices de style exposés dans l'article de Jacqueline Bossé-Andrieu (1988) : 
«Conception d'exercices de style dans l'optique de la traduction», Meta, 33-2, entre dans le cadre des exercices défini ici.

10. L'expression «types de discours» nous semble préférable à celle de «langues de spécialité» en ce qu'elle est respectueuse de l'unité de la langue. Il s'agit d'étudier une langue, le français, dans la diversité et la richesse de ses différents types de discours. Les langues de spécialité présupposent le morcellement de la langue d'après le cloisonnement de ses utilisateurs en fonction de critères socio-professionnels ou autres. Or, le traducteur. même quand il se spécialise, sait que la langue qu'il utilise fait partie d'un tout, d'une unité linguistique et culturelle individuelle.

11. Par corpus, il faut entendre un ensemble de discours constitué d'après les mêmes critères de collecte et soumis à la même méthode d'analyse, tels que l'ont défini J. Dubois et J. Sumpf (1969) in «Problèmes de l'analyse du discours», Langages, $\mathrm{n}^{\circ} 13$.

12. Malgré la similitude des termes, la transformation syntaxique envisagée ici ne relève pas de la grammaire générative puisqu'elle est le fait du locuteur, qui, indépendamment des contraintes linguistiques, choisit par exemple d'utiliser un passif plutôt qu'un actif, une nominalisation plutôt qu'une construction verbale. Elle a une valeur énonciative qu'il incombe au traducteur de réénoncer.

13. Pour faire prendre conscience aux apprentis-traducteurs de cette particularité du discours publicitaire, il a suffi de leur faire collecter des pages publicitaires des mêmes modèles de voiture dans la presse française et la presse israélienne. On ne vantera pas les mêmes performances de la Renault 19 dans le Figaro et dans Maariv.

14. Les hasards de l'actualité ont bien fait les choses: à la même époque, deux procès de criminels nazis, en France, celui de Barbie; en Israël, celui de Demanjuk, nous ont fourni un corpus qui a donné lieu à une analyse stylistique comparative.

15. Ce type de travail de recherche est essentiel pour la formation d'un traducteur. Il semble relever plus d'un enseignement de la civilisation que de celui de la stylistique. Et pourtant, si la stylistique est ce point qui relie la langue à la parole, n'est-il pas important pour notre apprenti-traducteur de savoir, de même qu'il sait que le possessif en hébreu intègre la relation au possesseur, ce que le français ne fait pas, que le mot 'ets en hébreu n'a pas le même champ lexical que le mot arbre car il désigne également la matière, le bois; ou encore, de même qu'il sait que l'expression idiomatique Mitat Sdom ne se traduira pas par «le lit de Sodome», mais par «le lit de Procuste», car les mythes n'ont pas la même origine dans les deux civilisations, ne lui est-il pas nécessaire de savoir que les deux grandes écoles de formation des hauts commis de l'État en France, l'ENA et Polytechnique, n'ont pas d'équivalents en Israël où les hauts fonctionnaires ont une formation essentiellement politique partisane. Il faudrait dire, en parodiant Térencc : Rien de ce qui est contexte, situation, culture, extra-linguistique n'est étranger à la stylistique. 